\title{
Cáncer de Cervix
}

\author{
Dres.: Néstor H. Amorocho*, María Cecilia Arturo R**. \\ HOSPITAL SAN JUAN DE DIOS DE CALI \\ $1983-1984$
}

\section{INTRODUCCION}

El cáncer de Cervix Uterino no es enfermedad nueva. Se había reconocido 2.500 años antes de Cristo como "una molestia femenina" y algunas recetas se prescribían para combatirla (13).

Corresponde a la mitad de los cánceres que afectan al sistema reproductivo femenino (9), incluyendo Mama y una tercera parte de los tumores que se presentan en mujeres en la ciudad de Cali (11).

Factores de comportamiento sexual como edad temprana al primer coito, parto temprano, multiparidad, promiscuidad sexual e infección viral, se han encontrado asociados estadísticamente con la enfermedad (13), (8).

La importancia de factor marital (sexual) en la génesis de la neoplasia de cervix fue dramáticamente demostrada por Gagnon, quien estudió 13.000 monjas canadienses y no encontró casos de cáncer cervical (10). Towne, en otro estudio, confirmó este hallazgo (21).

Jefe Servicio de Ginecología

R3 Gineco-Obstetricia - Univalle
La gran mayoría de muertes por cáncer de cervix ocurren en mujeres humildes, en edad procreativa y por lo general madres de varios niños, lo cual se agrava si se considera dentro de este mismo grupo de mujeres su inestabilidad conyugal (12).

Pacientes con bajo nivel socio-económico tienen una más alta prevalencia de neoplasia cervical que aquellas que están mejor económicamente (5), (6).

Un estudio sobre 34.318 pacientes en California (7), mostró que el riesgo de cáncer cervical había aumentado en mujeres que tenían menos de 40 años de edad. Sin embargo, la Tercera Encuesta Nacional de Cáncer en U.S.A. dio a conocer que la rata de edad específica para el cáncer cervical invasivo permanecía relativamente constante después de los 45 años, en mujeres blancas, pero continuaba creciendo en las negras (7).

La introducción de la citología en la práctica ginecológica, representa un cambio significativo en el descubrimiento del cáncer cervical que hoy se diagnostica en etapa más temprana, además de que permite el diagnóstico de sus antecesores, esto es, la displasia y el carcinoma in situ (20), (19), (17). 
Desde 1925, cuando Hinselman ideara la colposcopia, fue necesario que transcurrieran años para reconocer universalmente que tanto la citología como la colposcopia, tenían posibilidades y limitaciones en el diagnóstico del cáncer y que ambos métodos se complementan. Sin embargo, es difícil recurrir a ambos métodos, en gran escala, dada la falta de equipos y suficientes colposcopistas con experiencia (3). En la actualidad, la función principal de la colposcopia radica en la valoración clínica de pacientes con citología exfoliativa anormal (20), (15).

Foote y Stewart, investigaron en 1948 la localización anatómica del carcinoma epidermoide intra epitelial del cuello uterino. Las lesiones se encontraban en el orificio externo y en torno al mismo y a pesar de hallarlas con alguna regularidad en el trayecto endocervical, se ubicaban con más frecuencia en la porción vaginal del cuello (20). Diez años después, se confirmó que las lesiones del carcinoma cervical in situ afectan, en general, el orificio externo y la porción vaginal del cuello. Sólo $2 \%$ quedarán limitadas al conducto endocervical (16).

Una de las primeras contribuciones para mejorar la toma de biopsia de cervix, conocida inicialmente como no dirigida, "ciega", de los cuatro cuadrantes, fue aportada por Schiller, cuando indicó la "aplicación de yodo y raspado del epitelio de la porción vaginal del cuello", en 1929.

Utilizando la prueba de Schiller, como método para efectuar "biopsia dirigida" del cuello uterino, se ha señalado una exactitud del $84 \%$ al $96.9 \%$ para los casos de carcinoma in situ y de temprana invasión del estroma (14).

En instituciones equipadas para valorar todas las lesiones del cuello uterino, mediante biopsias dirigidas y raspado endocervical, la conización queda reservada para investigar el caso no usual (17), (20), (15), (18) y como procedimiento terapéutico. Su uso se recomienda en pacientes jóvenes, con lesiones localizadas y que puedan ser controladas adecuadamente.

Hace diez años se presentó al XI Congreso Colombiano de Obstetricia y Ginecología, por parte de uno de los autores, una revisión sobre carcinoma de cervix efectuada en el mismo hospital en 1973 (1) y posteriormente se publicó en la revista Colombiana de Obstetricia y Ginecología, el trabajo titulado "CARCINOMA IN SITU DE CERVIX-DIAGNOSTICO Y MANEJO-EIOPSIA FRENTE A CONO" (2).

Al releer estos artículos, sentimos la necesidad de investigar que sucedía en nuestro hospital después de diez años frente al diagnóstico de ca. de cervix y cuál podía ser la evolución en cuanto a diagnóstico, manejo y posibles perspectivas, consideraciones que nos llevaron a realizar el presente trabajo.

\section{MATERIAL Y METODOS}

Para el presente trabajo se hizo una revisión estadística de las historias de pacientes egresadas del Hospital de San Juan de Dios de Cali, con diagnóstico de ca. de cervix, durante el período comprendido entre el 1o. de enero de 1983 y el 31 de diciembre de 1984.

Se establece una comparación en cuanto a la frecuencia de esta patología, en diez años de evolución 1973,(1) a 1983.

A todas las pacientes se les había efectuado citología vaginal, rutina establecida en el servicio de Ginecología del hospital, previa a la consulta médica en consulta externa. 
Siguiendo las normas para el manejo de pacientes, se efectúa biopsia de cervix bajo Schiller a todas aquellas cuya citología sea informada grado III o mayor y se hace la valoración clínica respectiva con base al diagnóstico histopatológico de la biopsia que confirma el carcinoma.

Los estadios II y subsiguientes que hasta 1973 se diagnosticaron, fueron hospitalizados para completar su estudio y remisión posterior a otro centro hospitalario para cobaltoterapia. Desde 1974 hasta la actualidad, estas pacientes no se hospitalizan y su estudio se complementa a nivel de la consulta externa.

Aquellos casos diagriosticados como ca. in situ, siguiendo la conducta ya definida desde 1968, en el servicio de Ginecología del hospital San Juan de Dios de Cali (18), se llevan a histerectomía, sin efectuar conización previa a la intervención.

Se reserva el cono sólo para pacientes que no aceptan la extirpación del útero (4), por razones de edad y con miras a conservar la función obstétrica.

\section{Epidemiología - Incidencia}

Durante los dos años considerados para el presente estudio, se encontró que el total de egresos hospitalarios fue de 8.965 pacientes correspondientes a

Cuadro No. 1

HOSPITAL SAN JUAN DE DIOS 1973/74 - 1983/84 COMPARACION DE EGRESOS HOSPITALARIOS A 10 AÑOS

\begin{tabular}{|c|c|c|c|c|c|c|}
\hline$A \bar{N} O S$ & \multicolumn{2}{|c|}{ HOMBRES } & \multicolumn{2}{|c|}{ MU JERES } & \multirow{2}{*}{\multicolumn{2}{|c|}{\begin{tabular}{|c|c|} 
TOTALES & $\sigma \% x^{0}$ \\
No & $\%$ \\
\end{tabular}}} \\
\hline VARIABLE & No & $\%$ & No. & $\%$ & & \\
\hline 1973 & 2045 & 40.53 & 3.001 & 59.47 & 5.046 & 52.50 \\
\hline 1974 & 1.747 & 38.27 & 2.818 & 61.73 & 4.565 & 47.50 \\
\hline TOTALES & 3.792 & 39.45 & 5.819 & 60.54 & 9.611 & 100.00 \\
\hline
\end{tabular}

Ref.: REVISTA COLOMBIANA OBSTETRICIA Y GINECOLOGIA : Val. XXVIII; N.6 1.977

\begin{tabular}{|c|c|c|c|c|c|c|}
\hline 1983 & 1.903 & 43.16 & 2.506 & 56.84 & 4.409 & 49.18 \\
\hline 1984 & 1.929 & 42.34 & 2.627 & 57.66 & 4.556 & 50.82 \\
\hline TO TALES & 3.832 & 42.74 & 5.133 & 57.25 & 8.965 & 100.00 \\
\hline
\end{tabular}


4.409 en 1983 y 4.556 en 1984 . El mismo parámetro para los años 1973 y 1974 arroja un total de egresos hospitalarios de 9.611, correspondiente a 5.046 y 4.565 respectivamente en los años mencionados, para establecer comparación.

Al efectuar el análisis de egresos hospitalarios por sexo, encontramos que para las mujeres correspondió en 1973 a $3.001=59.47 \%$; en 1974 se encontró $2.818=61.73 \%$, para un total de $5.819=$ $60.54 \%$ casos en los dos años.

Diez años después los egresos femeninos correspondían a $2.506=56.84 \%$ para 1983 y $2.627=57.66 \%$ para 1984 , obteniéndose un total de $5.133=57.25 \%$ para los dos años (Cuadro No. 1).
Se observa en estas cifras que la demanda de servicios hospitalarios en la institución es mayor para mujeres que para hombres.

Téngase en cuenta que el hospital de San Juan de Dios de Cali no posee servicios de hospitalización en Pediatría ni Obstetricia. Como en trabajos anteriores publicados (1), (2), todavía se encuentra en período de remodelación de su planta física y el total de camas actual de 108 , es sensiblemente menor al considerado en 1973 y 1976, cuyo número era de 130.

Al efectuar el estudio de los diagnósticos de egresos, prosiguiendo en la búsqueda de cáncer, sin discriminar por sexo, se encontró para 1983 un total de 226 casos, $5.13 \%$.

Cuadro No. 2

HOSPITAL SAN JUAN DE DIOS

EGRESOS HOSPITALARIOS PACIENTES CON CANCER POR SEXO Y AÑO

COMPARACION $1973=6.77 \%$

\begin{tabular}{|c||c|c|c|c|}
\hline VARIABLE & H & $M$ & $\%$ & TOTAL \\
\hline 1983 & 82 & 144 & 5.13 & 226 \\
\hline 1984 & 70 & 136 & 4.52 & 206 \\
\hline TOTAL & 152 & 280 & 4.82 & 432 \\
\hline
\end{tabular}

\% CON LA POBLACION TOTAL EgRESADA. 
Para el año de 1984 el porcentaje de cáncer fue de $4.52 \%$ sobre los egresos hospitalarios, cifra absoluta 206 casos.

Para 1973, diez años atrás, se encontró una cifra porcentual de $6.77 \%$ sobre la población hospitalaria general. (Cuadro No. 2).

El cuadro No. 3, muestra la clasificación de tumores en hombres y mujeres para los años 1983 y 1984.

Para hombres tanto en 1983 como en 1984, la incidencia mayor se encuentra en los clasificados como gástricos y páncreas.

Para mujeres, la mayor concentración en ambos años se encuentra en mama. La mínima incidencia, sensiblemente igual para hombres y mujeres, se encuen- tra a nivel de los clasificados como óseo, esófago y piel.

\section{RESULTADOS}

\section{Cuadro No. 4:}

Haciendo abstracción de localizaciones a ambos sexos y dirigiéndonos específicamente a la mujer, encontramos que el cáncer femenino ocurre, según el estudio realizado por uno de los autores, en 1973 en $35.50 \%$ del total de casos con diagnóstico de cáncer. Haciendo consideración en relación porcentual, aisladamente sobre el total de cáncer femenino, 153 casos, vemos como el ca. de cervix pasa a ser $50.98 \%$, mama ocupa el segundo lugar con $39.87 \%$ y ovario se coloca en tercer lugar con $9.15 \%$.

Cuadro No. 3

\section{CLASIFICACION DE TUMORES}

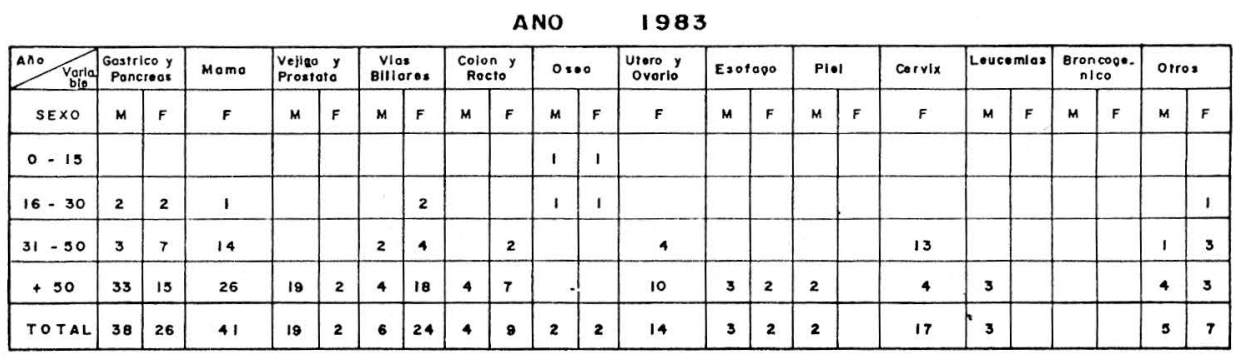

A N̄ O

1984

\begin{tabular}{|c|c|c|c|c|c|c|c|c|c|c|c|c|c|c|c|c|c|c|c|c|c|c|c|}
\hline \multirow{2}{*}{$\begin{array}{c}\text { Año } \begin{array}{c}\text { Vorla } \\
\text { blo }\end{array} \\
\text { SEXO }\end{array}$} & \multicolumn{2}{|c|}{$\begin{array}{l}\text { Gostrlco y } \\
\text { Poncreas }\end{array}$} & \multirow{2}{*}{$\frac{\text { Momo }}{F}$} & \multicolumn{2}{|c|}{$\begin{array}{l}\text { Velico y } \\
\text { Prostata }\end{array}$} & \multicolumn{2}{|c|}{$\begin{array}{l}\text { Viaz } \\
\text { Blllores }\end{array}$} & \multicolumn{2}{|c|}{$\begin{array}{l}\text { Colon } y \\
\text { Rect to }\end{array}$} & \multicolumn{2}{|c|}{0300} & \multirow{2}{*}{$\begin{array}{c}\begin{array}{c}\text { Utero y } \\
\text { Ovorlo }\end{array} \\
F\end{array}$} & \multicolumn{2}{|c|}{ Esotopo } & \multicolumn{2}{|c|}{ Plel } & \multirow{2}{*}{$\frac{\text { Corvix }}{F}$} & \multicolumn{2}{|c|}{ Leueermio } & \multicolumn{2}{|c|}{$\begin{array}{l}\text { Broncoos. } \\
\text { nico }\end{array}$} & \multicolumn{2}{|c|}{ otros } \\
\hline & $M$ & $F$ & & $M$ & $F$ & M & $F$ & $m$ & $F$ & M & $F$ & & M & $F$ & $m$ & $\mathbf{F}$ & & M & $F$ & M & $\boldsymbol{F}$ & M & $F$ \\
\hline $0-15$ & & & & & & & & & & & & & & & & & & & & & & & \\
\hline $16 \cdot 30$ & & & 2 & & & & & 1 & 2 & 1 & & & & & & & 1 & & & & & & 2 \\
\hline $31-50$ & 8 & 6 & 12 & & & 1 & 6 & 1 & 1 & & 2 & 2 & & & 1 & 1 & 11 & 2 & 2 & 5 & 2 & & 1 \\
\hline+50 & 24 & 25 & 18 & 13 & 2 & 4 & 12 & - & 9 & & & 12 & 2 & $2^{\circ}$ & & & 2 & 2 & & & & 5 & 1 \\
\hline TOTAL & 32 & 31 & 32 & 13 & 2 & 5 & 18 & 2 & 12 & 1 & 2 & 14 & 2 & 2 & 1 & 1 & 14 & 4 & 2 & 5 & 2 & 5 & 4 \\
\hline
\end{tabular}


Cuadro No. 1 - 4

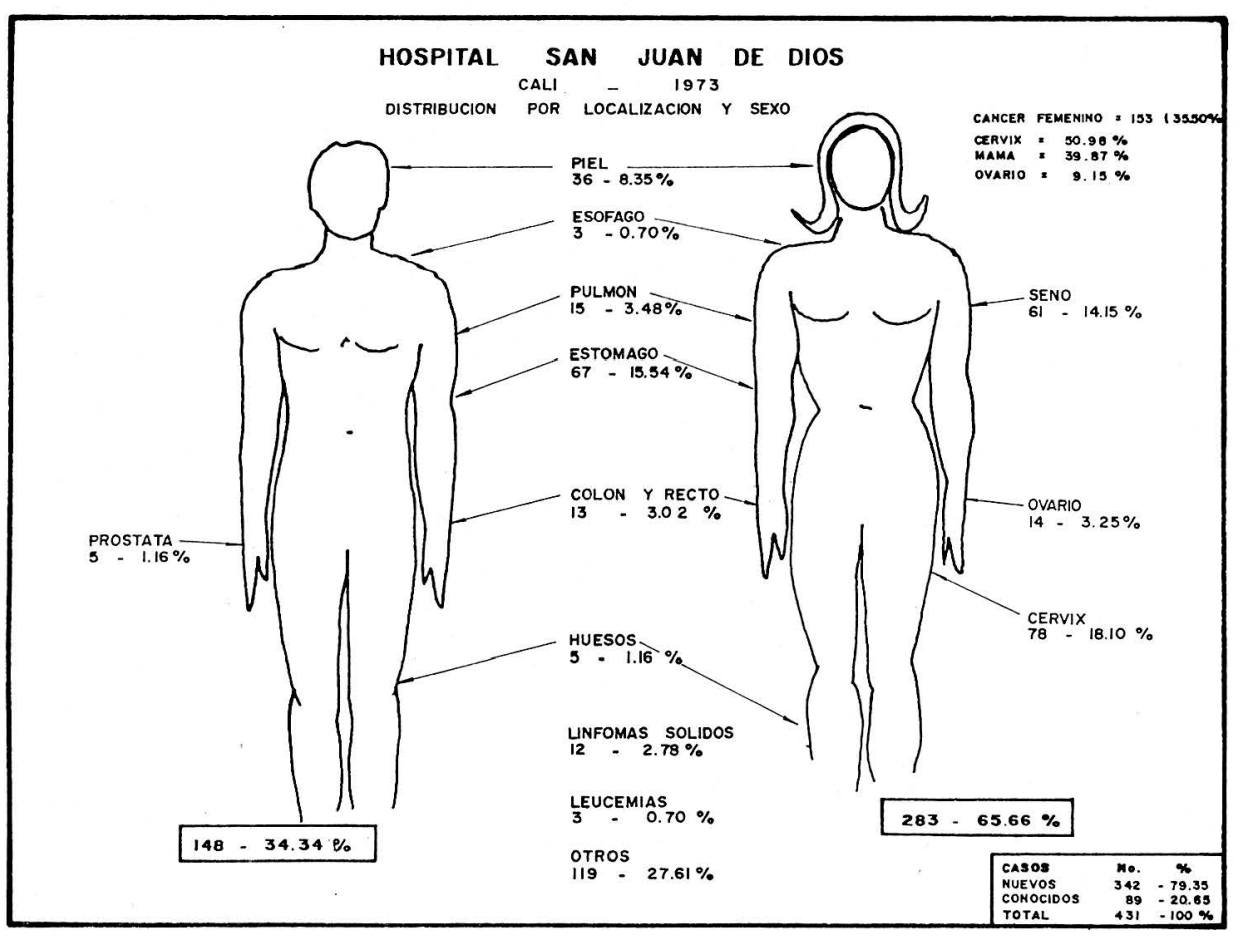

\section{Cuadros Nos. 5 y 6 :}

Efectuando idéntico análisis en 1983 y 1984 sobre cáncer femenino y estableciendo la relación porcentual que corresponde como se muestra en el cuadro No. 7, obtenemos totales para los dos años: mama $54.55 \%$, cervix $23.48 \%$ y útero-ovario $21.21 \%$.

Podemos entonces concluir que el ca. de cervix ocupa ahora el segundo lugar de frecuencia en la incidencia del cáncer femenino, perteneciendo el primer puesto al cáncer de mama que corresponde a la mitad de los casos de ca. para la mujer y el ca. de cervix sigue ocupando el primer lugar en el área genital propiamente dicha.
Cuadro No. 8:

Ahondando en nuestra investigación retrospectiva sobre ca. de cervix, encontramos que la incidencia porcentual sobre el total bruto de egresos hospitalarios va de una cifra de $0.81 \%$ - tasa bruta $8.1 \%$ - en 1974 , a $0.30 \%$-tasa bruta $3.0 \%$ - en 1984 .

Analizados los años 1973 y 1983 sobre el mismo total bruto de egresos hospitalarios, las cifras corresponden a $0.63 \%$ -tasa bruta $6.3 \%$ o- y $0.38 \%$ para 1983 -tasa bruta $3.8 \%$ -

Los totales para los años considerados al principio y final de la década, nos muestran una disminución de ca. de 
Cuadro No. 5

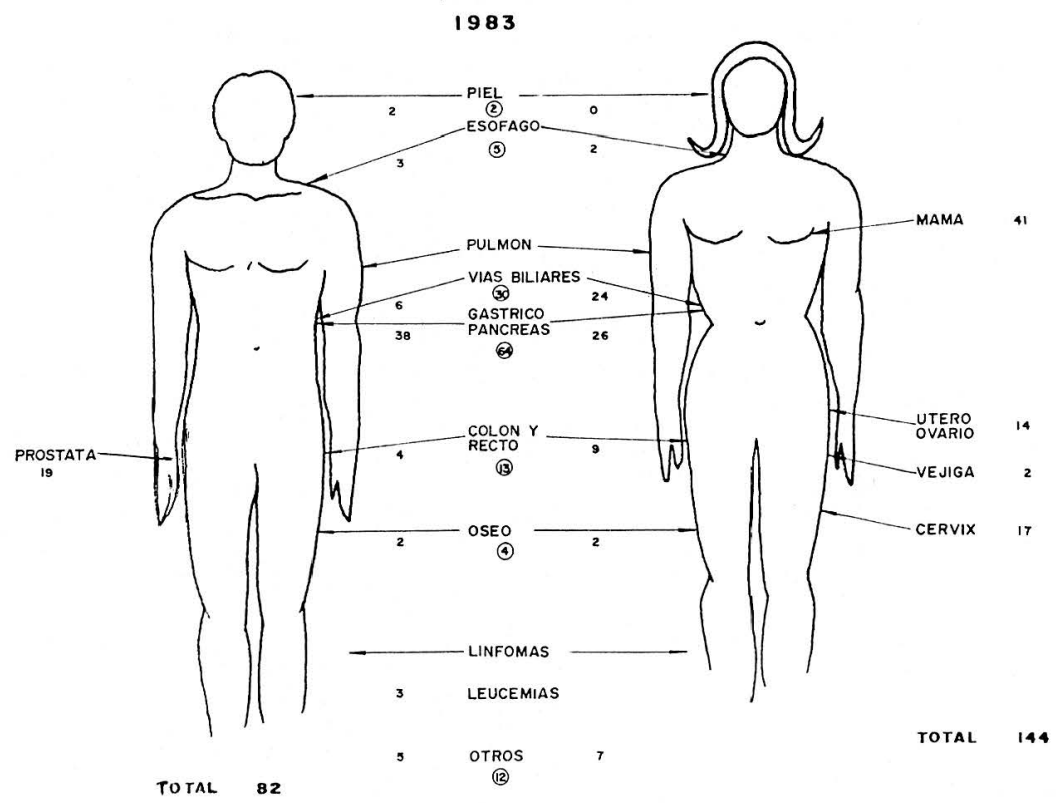

\section{Cuadro No. 6}

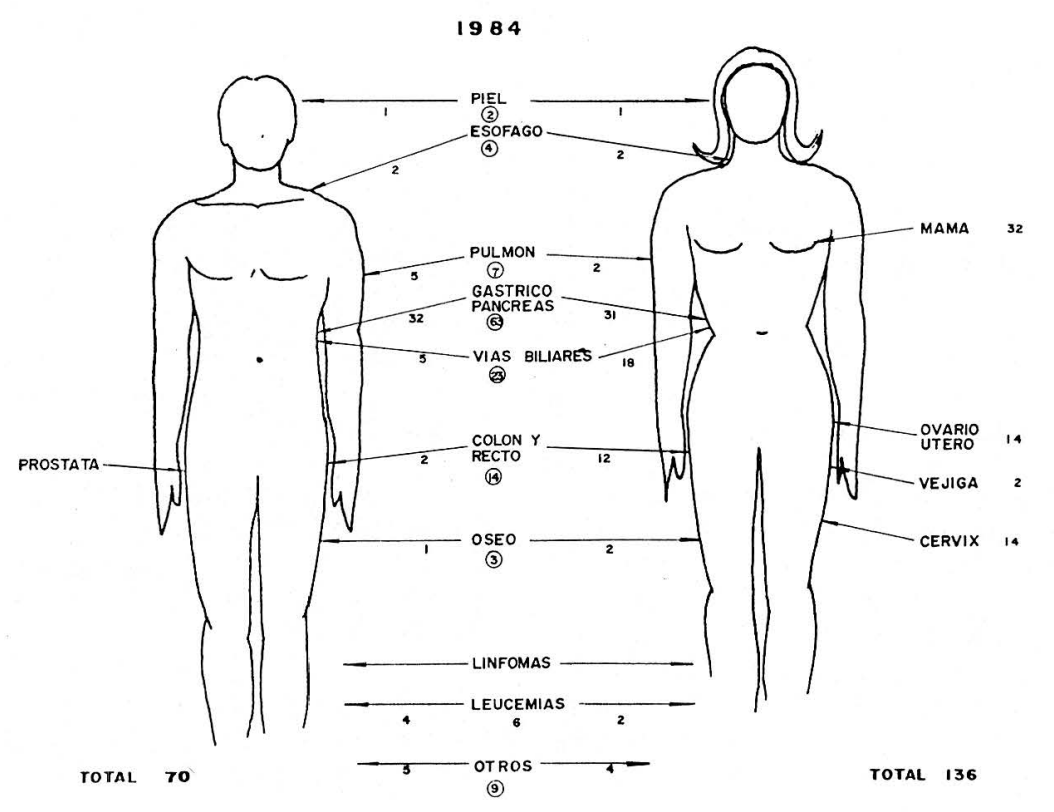


Cuadro No. 7

CANCER FEMENINO RELACION PORCENTUAL COMPARACION $1973-1983$ y 1984

\begin{tabular}{|c|c|c|c|c|c|c|c|c|}
\hline & CAHCE R & FE ME NINO & \multicolumn{2}{|c|}{ MAMA } & \multicolumn{2}{|c|}{ UTERO - OVARIO } & \multicolumn{2}{|c|}{ CERVIX } \\
\hline VARIABLE & $\mathrm{N}^{\circ}$ & $\%$ & $\mathbf{N}^{\circ}$. & $\%$ & No. & $\%$ & $\mathrm{No}$. & $\%$ \\
\hline 1973 & 153 & 35.50 & 61 & 39.87 & 14 & 9.15 & 78 & 50.98 \\
\hline
\end{tabular}

Ref. : AMOROCHO, N.; $X I$ CONGRESO COL. OBSTETRICIA $Y$ GINECOLOGIA BARRANQUILLA 1975

\begin{tabular}{|c|c|c|c|c|c|c|c|c|}
\hline 1983 & 72 & 31.85 & 41 & 56.94 & 14 & 19.44 & 17 & 23.61 \\
\hline 1984 & 60 & 29.13 & 32 & 53.33 & 14 & 23.33 & 14 & 23.33 \\
\hline TOTAL & 132 & 30.55 & 72 & 54.55 & 28 & 21.21 & 31 & 23.48 \\
\hline
\end{tabular}

Cuadro No. 8

EGRESOS CA. DE CERVIX Vs. EGRESOS HOSPITALARIOS TOTALES

$$
\begin{array}{r}
1983-1984 \\
1973-1974
\end{array}
$$

\begin{tabular}{|c|c|c|c|c|}
\hline \multicolumn{2}{|c|}{ EGRESOS } & \multicolumn{2}{c|}{ CA. } & CERVIX (0-IV) \\
\hline VARIABLE & No. & $\%$ & $\%$ \\
\hline $1973 *$ & $5.046 *$ & & $32 *$ & $0.63 *$ \\
1983 & 4.408 & 100 & 17 & 0.38 \\
\hline $1974 *$ & $4.565 *$ & & $37 *$ & $0.81 *$ \\
\hline 1984 & 4.556 & 100 & 14 & 0.30 \\
\hline & $9.611 *$ & & $69 *$ & 0.72 \\
\hline
\end{tabular}


cervix que va de $7.2 \%$ o para $1973 / 1974$, hasta $3.5 \%$ on $1983 / 1984$, considerados los egresos hospitalarios totales en los mismos períodos bianuales.

\section{Cuadro No. 9:}

Las cifras anotadas establecen una relación porcentual significativa, cuando las comparamos con el total de egresos de pacientes con cáncer, para los años considerados de 1983 y 1984, donde al sumar los dos años obtenemos $7.18 \%$ para un egreso por cáncer de 432 casos.

En los mismos años, 1983/1984 el total de egresos de mujeres con cáncer fue de 280 casos y de ellos, 31 fueron calificados como ca. de cervix estadios 0 a IV.

Concluimos entonces que para el período de 1983/1984, el ca. de cervix, sin considerar su clasificación por esta- dío, correspondió al $11.07 \%$ del total de mujeres hospitalizadas, que a su egreso tuvieron diagnóstico de cáncer.

\section{Cuadros Nos 10 y 11:}

Se estableció la relación ca. de cervix in situ frente a otros estadios y se compara con los hallazgos de 1973 y 1974.

Hacemos notar la disminución apreciable tanto en los estadíos 1-IV, como en ca. de cervix in situ en el lapso de diez años. Esto indica una baja franca en la incidencia de ca. de cervix 1-IV y una disminución significativa de ca. de cervix in situ, lo cual estaría muy probablemente en favor de un aumento en el diagnóstico de las displasias.

\section{Cuadro No. 12:}

Nos muestra la correlación citologíabiopsia-patología post-quirúrgica.

Cuadro No. 9

EGRESOS HOSPITALARIOS CA. CERVIX

VS. TOTAL DE

EGRESOS HOSPITALARIOS PTES. CON CANCER

\begin{tabular}{|c|c|c|c|c|c|c|}
\hline$A \overline{N O}$ & EGRESOS PTES & CON CANCEF & EGRESOS & $\begin{array}{l}\text { PTES } \\
10-\end{array}$ & $\begin{array}{l}\operatorname{con} \\
|V|\end{array}$ & CA. CERVIX \\
\hline VARIABL & $\mathrm{N}^{\circ}$. & $\%$ & $\mathrm{~N}^{\circ}$. & & & $\%$ \\
\hline 1933 & 226 & 100 & 17 & & & 7.52 \\
\hline 1934 & 206 & 100 & 14 & & & 6. 79 \\
\hline TOIAL & 432 & - & 31 & & & 7. 18 \\
\hline
\end{tabular}


Cuadro No. 10

RELACION CA. CERVIX IN SITU FRENTE A OTROS ESTADIOS POR AÑOS

\begin{tabular}{|c|c|c|c|c|c|}
\hline \multicolumn{1}{|c|}{ AÑO } & CA. CERVIX & $\%$ & $1-I V$ & $\%$ & TO TAL \\
\hline 1970 & 18 & 43.90 & 23 & 56.10 & 41 \\
\hline 1971 & 15 & 40.54 & 22 & 59.46 & 37 \\
\hline 1972 & 11 & 37.93 & 18 & 62.07 & 29 \\
\hline 1973 & 20 & 62.50 & 12 & 37.50 & 32 \\
\hline 1974 & 18 & 48.63 & 19 & 51.35 & 37 \\
\hline 1975 & 25 & 65.79 & 13 & 34.21 & 38 \\
\hline 1976 & 12 & 80.00 & 3 & 20.00 & 15 \\
\hline
\end{tabular}

REF.: REVISTA COLOMBIANA OBSTETRICIA Y GINECOLOGIA: VOL. XXVIII: No 6 1977

Cuadro No. 11

COMPARACION DE PATOLOGIA CERVICAL A 10 AÑOS

\begin{tabular}{|c|c|c|c|c|c|c|}
\hline & IN & & I & - IV & T 0 & \\
\hline 1973 & 20 & \multirow{2}{*}{38} & 12 & \multirow{2}{*}{31} & 32 & \multirow{2}{*}{69} \\
\hline 1974 & 18 & & 19 & & 37 & \\
\hline 1983 & 13 & \multirow{2}{*}{26} & 4 & \multirow{2}{*}{5} & 17 & \multirow{2}{*}{31} \\
\hline 1984 & 13 & & 1 & & 14 & \\
\hline
\end{tabular}

Si tomamos para efecto de la conducta quirúrgica, que se sigue en nuestro hospital, como correctos los diagnósticos por biopsia de ca. in situ: $22=38.60 \%$ y displasia: $28=49.12 \%$ y asimilamos en el mismo sentido la cervicitis: $1=1.75 \%$,
Cuadro No. 12

1983 - 1984 RELACION PORCENTUAL CITOLOGIAS Vs BIOPSIAS VS PATOLOGIAS

\begin{tabular}{|c|c|c|c|c|c|c|c|c|}
\hline CITOLOGIAS & \multicolumn{4}{|c|}{ BIOPSIAS } & \multicolumn{4}{|c|}{ PATOLOGIA OX. } \\
\hline & cenvicitis & OSPRLASLOS & $\begin{array}{c}\text { C.A. } \\
\text { IMSITU }\end{array}$ & \begin{tabular}{|c|} 
C. $\mathbf{A}$. \\
INVABSO
\end{tabular} & CERVItaris & Displasios & . & \begin{tabular}{|c|}
$C . A$ \\
INVASIVO
\end{tabular} \\
\hline $\begin{array}{l}\text { ATIPICAS } \\
\text { (IIII) }\end{array}$ & 1 & 19 & 9 & 2 & 2 & 17 & 11 & 1 \\
\hline $\begin{array}{l}31 \\
54.39 \%\end{array}$ & (1.75) & $(33.33)$ & $(15.80)$ & (3.5) & $(3.56)$ & $(29.82)$ & (19.29) & $(1.75)$ \\
\hline $\begin{array}{c}\text { IV }-v \\
26 \\
45.61 \%\end{array}$ & - & $\begin{array}{c}9 \\
(15.79)\end{array}$ & $\begin{array}{c}13 \\
(22.80)\end{array}$ & $\begin{array}{c}4 \\
(7.0)\end{array}$ & $\begin{array}{c}1 \\
(1.75)\end{array}$ & $\begin{array}{c}6 \\
(10.52)\end{array}$ & $\begin{array}{c}15 \\
(26.31)\end{array}$ & $\begin{array}{c}4 \\
(7.0)\end{array}$ \\
\hline $\begin{array}{r}\text { TOTAL } 57 \\
100 \%\end{array}$ & $\begin{array}{c}1 \\
1.75 \%\end{array}$ & $\begin{array}{c}28 \\
49.12 \%\end{array}$ & $\begin{array}{l}22 \\
33.6 \%\end{array}$ & $\begin{array}{c}6 \\
10.50^{\circ}\end{array}$ & $\begin{array}{c}3 \\
5.26 \%\end{array}$ & \begin{tabular}{|c|}
23 \\
$40.34 \%$
\end{tabular} & $\begin{array}{l}26 \\
45.618\end{array}$ & $\begin{array}{c}5 \\
8.78 \%\end{array}$ \\
\hline
\end{tabular}

obtenemos un total de 51 biopsias = $89.47 \%$.

Si por otro lado, tomamos los diagnósticos post-quirúrgicos ca. in situ: $26=$ $45.61 \%$, displasias: $23=40.35 \%$ y cervicitis: $3=5.26 \%$, obtenemos un total de 52 piezas quirúrgicas diagnosticadas $=$ $91.22 \%$. 
La diferencia entre los dos factores, diagnóstico por biopsia y confirmación diagnóstica de la pieza quirúrgica, ofrece un desfase de $1.75 \%$. Un caso diagnosticado por la biopsia como invasivo no comprobado por el estudio de la pieza quirúrgica. Como conclusión, al establecer comparación con trabajo anterior (2) cuando se encontró un error en contra de la conducta quirúrgica seguida de $4.2 \%$, en los siguientes diez años se observa mejoría apreciable y un margen de error de $1.75 \%$ en este caso a favor, nos confirma la tesis planteada en 1975 de omitir la conización, como paso previo para confirmar el diagnóstico y llevar la paciente a cirugía (2).

\section{Cuadro No. 13:}

Se trata de establecer la relación porcentual de las citologías frente a los subsiguientes pasos, esto es, biopsia y cirugía.
Se establecieron dos grupos de citologías: 31 correspondientes a grado III y 26 a grados IV $-\mathrm{V}$.

Analizando la relación biopsia-patología quirúrgica y correlacionando la citología que motivó el proceso, se observa que la mayor concentración para ca. invasivo está en las citologías grados IV - V; sigue en su orden decreciente el ca. in situ y la displasia.

El grupo de citologías III muestra valores numéricos y porcentuales inversos, tanto en la biopsia como en la patología quirúrgica, observándose la mayor concentración en las displasias disminuyendo para el ca. in situ y poco significativas para el ca. invasivo.

En conclusión: citologías atípicas, grado III son tributarias en su orden numérico y porcentual de displasia ca. in situ y ca. invasivo; citolog ías grados IV -

Cuadro No. 13

CORRELACION CITOLOGIA - BIOPSIA - PATOLOGIA POST. OX.

$1983-1984$

\begin{tabular}{|c|c|c|c|c|c|c|c|c|c|c|c|c|}
\hline \multirow{3}{*}{$\begin{array}{c}\text { CITOLOGIAS } \\
\text { ATIPICAS } \\
\text { (III). }\end{array}$} & \multicolumn{6}{|c|}{ BIOPSIAS } & \multicolumn{6}{|c|}{ PATOLOGIAS } \\
\hline & \multirow[b]{2}{*}{$\begin{array}{c}\text { CERVICI- } \\
\text { YIS }\end{array}$} & \multicolumn{3}{|c|}{ DISPLASIAS } & \multirow{2}{*}{\begin{tabular}{|c|} 
CA. \\
IN SITU
\end{tabular}} & \multirow{2}{*}{$\begin{array}{c}\text { CA. } \\
\text { INVASIVO }\end{array}$} & \multirow[b]{2}{*}{$\begin{array}{c}\text { CERyICI- } \\
\text { TIS }\end{array}$} & \multicolumn{3}{|c|}{ DISPLASIAS } & \multirow{2}{*}{\begin{tabular}{|c|} 
CA. \\
IN SITU \\
\end{tabular}} & \multirow{2}{*}{$\begin{array}{c}\text { CA. } \\
\text { INVASIVO }\end{array}$} \\
\hline & & LEVE & MOOERADA & SEVERA & & & & LEVE & MODERADA & SEVERA & & \\
\hline 31 & 1 & 4 & 5 & 10 & 9 & 2 & 2 & 2 & 8 & 7 & 11 & 1 \\
\hline $54.39 \%$ & $3.2 \%$ & \multicolumn{3}{|c|}{$61.29 \%$} & $29.03 \%$ & $6.45 \%$ & $6.45 \%$ & $6.45 \%$ & $25.8 \%$ & $22.58 \%$ & $35.48 \%$ & $3.22 \%$ \\
\hline \multicolumn{13}{|l|}{ IV $y$ V } \\
\hline 26 & - & - & 6 & 3 & 13 & 4 & 1 & - & 3 & 3 & 15 & 4 \\
\hline $45.61 \%$ & & \multicolumn{3}{|c|}{$34.6 \%$} & $50 \%$ & $15.3 \%$ & $3.8 \%$ & \multicolumn{3}{|c|}{$2300 \%$} & $57.69 \%$ & 15.38 \\
\hline $\begin{array}{r}\text { TOTAL } \\
57\end{array}$ & I & 4 & II & 13 & 22 & 6 & 3 & 2 & 11 & 10 & 26 & 5 \\
\hline
\end{tabular}


Cuadro No. 14

CITOLOGIAS LUGAR PROCEDENCIA

$1983-1984$

\begin{tabular}{|l||cc|cc|}
\hline \multirow{2}{*}{ H.S. J. D. } & \multicolumn{2}{|c|}{1983} & \multicolumn{2}{c|}{1984} \\
\cline { 2 - 5 } & 7 & $12.28 \%$ & 2 & $3.5 \%$ \\
\hline C. De SALU & 20 & $35.08 \%$ & 20 & $35.08 \%$ \\
\hline & & & 1 & $1.75 \%$ \\
\hline UNICANCER & 2 & $3.5 \%$ & 1 & $1.75 \%$ \\
\hline OTROS & 4 & $7.0 \%$ & 1 & \\
\hline
\end{tabular}

$V$, siguiendo el mismo orden, son tributarias de ca. invasivo, ca. in situ y displasia.

Por lo tanto, creemos que toda citología desde grado III debe conducir a biopsia de cervix, ojalá dirigida por colposcopia o por lo menos, como se hace de rutina en nuestro hospital, bajo prueba de Schiller.

Cuadro No. 14:

Muestra el lugar de procedencia de las 57 citologías analizadas en 1983 y 1984 fuente de universo del trabajo, para encontrar los casos con diagnósticos de ca. de cervix.

El $70.16 \%$ de todas las citologías estudiadas procede de Centros de Salud del Municipio de Cali. 9\% de las citologías corresponden a pacientes que consultaron al Centro Hospitalario; $5 \%$ a otros clasificados como médicos y laboratorios privados y un $3 \%$ remitidas de Unicáncer.

Se demuestra en este análisis que el hospital de San Juan de Dios de Cali, funciona como centro de referencia para el Sistema del Servicio de Salud del

Cuadro No. 15

1983 - 1984 DISTRIBUCION POR EDAD

\begin{tabular}{|c|c|c|c|c|c|c|c|c|}
\hline \multirow{2}{*}{$15-19$} & \multicolumn{2}{|c|}{ CERVICITIS } & \multicolumn{2}{c|}{ DIS PLASIA } & \multicolumn{2}{c|}{ CA. IN SITU } & \multicolumn{2}{c|}{ CA. INVASIVO } \\
\cline { 2 - 9 } & 1983 & 1984 & 1983 & 1994 & 1983 & 1984 & 1983 & 1994 \\
\hline $20-29$ & & & & & & & & \\
\hline $30-39 *$ & 1 & 1 & 7 & 3 & 7 & 8 & - & 1 \\
\hline $40-49 *$ & 1 & - & 4 & 1 & 3 & 2 & 3 & - \\
\hline $50-59$ & - & - & 4 & 2 & 2 & 2 & - & - \\
\hline $60+$ & - & - & & 1 & 1 & - & 1 & - \\
\hline TO TAL & 2 & 1 & 15 & 8 & 13 & 13 & 4 & 1 \\
\hline 57 Ptes. & $3.51 \%$ & $1.75 \%$ & $26.3 \%$ & $14.03 \%$ & $22.8 \%$ & $22.8 \%$ & $7.0 \%$ & $1.75 \%$ \\
\hline
\end{tabular}


Valle del Cauca, según norma del Sistema Nacional de Salud.

\section{Cuadro No. 15:}

Se aprecia que la distribución por edad centraliza la mayor incidencia de ca. de cervix, sin considerar el estadío, grupo que corresponde de los 30 a los 49 años.

Si nos fijamos detenidamente en los diagnósticos de ca. in situ, vemos que para ambos ar̃os 1983 y 1984, el mayor número se agrupa en las edades de 30 a 39 años y el ca. invasivo en las edades mayores, entre 40 y 49 años. Observación igual se hizo en trabajo anterior (2).

Vemos como la displasia ocupa el $36.84 \%-21$ casos concentrados en las mismas edades y por encima de ellasyrupo de 30 a 59 años, lo que está en favor de un diagnóstico precoz con base en la citología vaginal.

\section{Cuadro No. 16:}

Seguimiento de casos con citología III o mayor, controles por año, sobre el total de 57 citologías tributarias de los casos diagnosticados como ca. de cervix.

Nos muestra que una cuarta parte de las pacientes tienen de 1 a 2 controles por 24 meses. Téngase en cuenta que la fecha límite del estudio de diciembre 31 de 1984 no permite, según el análisis, considerar el seguimiento mayor de casos diagnosticados en el segundo semestre de 1984.

\section{Cuadro No. 17:}

La paridad, como en otros estudios, nos muestra que la patología cervical, en base a la citología, tiene relación directa con el mayor número de hijos y por ende, con mayor actividad sexual de las
Cuadro No. 16

No. DE CONTROLES POST. QX

\begin{tabular}{|c|c|c|c|c|}
\hline \multirow[b]{2}{*}{$-1 / A \bar{N} O$} & \multicolumn{2}{|c|}{1983} & \multicolumn{2}{|c|}{1984} \\
\hline & 1 & $1.75 \%$ & 9 & $15.78 \%$ \\
\hline $1-2 / A \overline{N O}$ & 12 & $21.05 \%$ & 13 & $22.80 \%$ \\
\hline $3+/ A \overline{N O}$ & 16 & $28.07 \%$ & 3 & $5.26 \%$ \\
\hline
\end{tabular}

Cuadro No. 17

\section{CORRELACION CITOLOGIAS Vs. PARIDAD}

\begin{tabular}{|c|ccc|c|}
\hline \multirow{2}{*}{$\leq 0$} & \multicolumn{3}{|c|}{$N^{\circ}$} & $\%$ \\
\cline { 2 - 5 } & 111 & 1 & 1 & 1.76 \\
\hline $1-3$ & 111 & 12 & 19 & 33.33 \\
\hline $4+$ & $1 V-V$ & 7 & 19 \\
\hline & $1 V-V$ & 10 & 37 & 64.91 \\
\hline
\end{tabular}

pacientes. Citologias III, IV y V se encontraron en $33.33 \%$ en pacientes con paridad hasta 3 y se duplica la cifra porcentual, $64.91 \%$, para pacientes con paridad 4 y mayor.

\section{RESUMEN}

1. Se presenta la incidencia total de cáncer para los años 1983-1984 en el hospital San Juan de Dios de Cali, Colombia, clasificando los casos por sexo y órganos o sistemas afectados.

2. Se establece comparación con similar trabajo realizado por uno de los autores diez años atrás, 1973-1974, en el mismo centro hospitalario.

3. Se encontró que el cáncer de cervix, sin considerar estadio, ocupa el segundo lugar de frecuencia en la incidencia de cáncer femenino para los años 1983 y 1984 y el primer lugar en el 
área genital propiamente dicha, $23.48 \%$ para los mismos años.

4. Se encontró una baja franca en la incidencia de ca. de cervix I - IV frente a los hallazgos diez años atrás (1973) y en el mismo lapso, disminución significativa de ca. de cervix in situ, en favor de aumento en el diagnóstico de displasia.

5. La conducta quirúrgica seguida en el hospital de San Juan de Dios de Cali, omitiendo la conización de rutina, en el manejo del ca. de cervix in situ, para confirmar el diagnóstico y llevar la paciente a cirugía, sigue siendo un buen procedimiento y sustenta la tesis planteada en 1975, puesto que el margen de error prácticamente no existe ahora.

6. No se desconoce el valor de la colposcopia para hacer biopsia dirigida en casos de citología III o mayor. La falta de recurso humano $y$ equipo en este campo no permite la secuencia lógica: citología-biopsia dirigida por colposcopia, conización, diagnóstico, trata- miento. En cambio se sigue usando el Test de Schiller con buenos resultados. Creemos que la pericia de nuestros Patólogos-Citólogos, es suficientemente confiable para decidir la conducta planteada.

7. El hospital de San Juan de Dios de Cali, funciona como centro de referencia, siguiendo las normas del Servicio de Salud del Valle y la concentración de citologías vaginales grado III a V $(70.16 \%)$, procedentes de Centros de Salud, demuestra un seguimiento adecuado de los casos en el programa de detección de cáncer cervical uterino.

8. Los parámetros Edad-Paridad, de nuestras pacientes frente a la incidencia de neoplasia intraepitelial, ca. de cervix in situ y ca. de cervix invasor, no difieren de los encontrados por otros autores.

9. La citología vaginal, más difundida ahora y una mayor concientización de las mujeres para hacer este examen periódico, es el mejor método para la detección precoz de patología cervical.

\section{CANCER OF THE UTERINE CERVIX}

\section{SUMMARY}

1. The total cancer incidence for years 1983-1984 at the Hospital San Juan de Dios, Cali, Colombia is presented, clasifying the cases by sex and organ or systems affected.

2. A comparisson is established with a simmilar work done by one of the authors ten years ago (1973-1974) at the same Hospital.

3. It was found that the cervix carcinoma, without taking in account the hospitalary lenght of stay, occupies the second place in frecuency of the incidence of female gynecological cancer for years 1983-1984, and the first place at the genital area, $23.48 \%$ for the same period of time.

4. It was found a dramatic decrease in the incidence of cervix carcinoma stages I-IV compared with the results found ten years ago (1973), and in the same period of time, a significant decrease in the diagnosis of in situ cervix carcinoma with an increase in the diagnosis of hyperplasia. 
5. The surgical management followed at the Hospital San Juan de Dios at Cali, excluding rutine conization for the management of in situ cervix carcinoma to confirm the diagnosis and take the patient to surgery, is still a good procedure and asserts the thesis pointed out in 1975 as the possibility for mistake does not exist by now.

6. The value of colposcopy for directed biopsies in cases of PAP III cytology or higher, is not ignored, but the lack of human resources and equipment in this field, does not allow a logic sequence: Cytology, colposcopy directed biopsy, conization, diagnosis and treatment. Instead, the Schiller test is still been used with good results. We think that the experience of our cytologists and pathologists is reliable enough to decide the proposed management.

\section{BIBLIOGRAFIA}

1. AMOROCHO N., HENAO F.,: Carcinoma de Cervix en el Hospital de San Juan de Dios de Cali. XI Congreso Colombiano de Obstetricia y Ginecología, Barranquilla. 1975.

2. AMOROCHO N., HENAO F.,: Carcinoma In Situ de Cervix. Diagnóstico y manejo. Biopsia frente a cono. Hospital de San Juan de Dios de Cali. Revista Colombiana de Obstetricia y Ginecología. Vol. XVIII No. 6., noviembre-diciembre 1977.

3. CARDONA F., ACEVEDO G., QUIJANO H., PARDO Francisco: Diagnóstico y Tratamiento de las Neoplasias Interepiteliales y de los Carcinomas Cervicales Invasores en el Embarazo. Revista Colomuiana de Obstetricia y Ginecología. Vol. XXXIV, No. 4, 1983.

4. CHRISTOPHERSON W., GRAY L.: Dysplasia and Preclinical Carcinoma of the Uterine Cervix: Diagnosis and Ma-
7. The Hospital San Juan de Dios at Cali, operates as a referal center and follows the standards of the Valle's health service and the concentration of vaginal citologies grades 111 to $V(70.16 \%)$ from our health center show and adequate follow up for the cases in the program for screening of cervical carcinoma.

8. The parameters age- parity of our patients compared with the cervical intraepithelial neoplasia, in situ cervix carcinoma and invasive cervical cancer incidences do not differ to those found by other authors.

9. Vaginal cytology, more spreaded now and a better motivation to women for a periodic examination is the best screening method for the detection of cervical pathology.

nagement. Seminars in Oncology. Vol. 9, No. 3. 1982.

5. CLEMMESEN J., NIELSEN A.,: The Social Distribution of Cancer in Copenhagen, 1943-1947. Brit J. Cancer 5: 159-171, 1951 .

6. DEVESA S.S., DIAMOND E.L., Association of Breast Cancer and Cervical Cancer Incidences with Income and Education Among Whites and Blacks. J. Nat Cancer Inst. 65: 515-528, 1980.

7. FASAL E., SIMMONS M.E., KAMPERT J.B.; Factors Associated with High and Low Risk of Cervical Neoplasia. J. Nat. Cancer Inst. 66: 613-636, 1981.

8. FENOGLIO C., FERENCYZ A.,: Etiologic Factors in Cervical Neoplasia. Seminars in Oncology. Vol. 9 No. 3, 1982.

9. GAITAN YANGUAS M., JORDAN G.,: Boletín Instituto Nacional de Cancero- 
logía. Vol. 2, No. 6. Bogotá, Colombia, 1974.

10. GAGNON F.,: Contributions to the Study of the Etiology and Prevention of Cervical Concer. Am. J. Obstet Gynecol. 60: 516-522, 1950.

11. GUZMAN N., BUENO M.,: Tendencia del Cáncer de Cervix Uterino en Cali. $\mathrm{X}$ Congreso Colombiano de Obstetricia y Ginecología, Cali, Colombia, 1973.

12. GUZMAN N., GUERRERO R.,: Cáncer de Cervix Uterino en Cali, Colombia. Revista Antioquia Médica. Vol. 21, 1971.

13. GUZMAN N.,: Cáncer de Cervix Uterino Histoepidemiología de una Enfermedad Social. Universidad del Valle, Facultad de Salud, Departamento de Medicina Social, Cali 1984.

14. GRIFFTIHS C., YOUNGE P.,: Punch Biopsy of the Cervix. Am. J. Obst. Gynecol. 88, 1964.

15. JORDAN G. y Cols.: Avances de el Manejo de las Neoplasias Cervicales Intraepiteliales. X Congreso Colombiano de Obstetricia y Ginecología. Cali, Colombia, 1973.
16. PRYZBORA L., PLUTOWA A.,: Cit. por STAFL. A. FRIEDRICH JR, E., MATTINGLY, R.: Diagnóstico de Neoplasia Cervical-Reducción del Riesgo de Error. Clínica Obstetricia y Ginecología Edit. Interamericana, junio 1973.

17. PULIDO A.,: Diagnóstico Citopatológico de las Lesiones Premalignas y Malignas del Cuello Uterino. Revista Colombiana Obstetricia y Ginecología. Vol. XX, No. 5, 1969.

18. RESTREPO H.; Correlación Cito-Histológica en Displasia y Ca. In Situ. X Congreso Colombiano de Obstetricia y Ginecología, Cali, Colombia 1973.

19. SPJUT H., FACHNER R.,: Diagnóstico Citológico de la Displasia y Carcinoma In Situ del Cuello Uterino. Clínica Obstetricia y Ginecología. Edit. Interamericana, Diciembre 1967.

20. STALF A., FRIEDRICH JR. E., MATTINGLY R.,: Diagnóstico de Neoplasia Cervical-Reducción del Riesgo de Error. Clínica Obstetricia y Ginecología. Edit. Interamericana, Junio 1973.

21. TOWNE J.E.,: Carcinoma of the Cervix in Nulliparous and Celebate Women. Am. J. Obst. Gynecol. 69: 606-613, 1955. 